\title{
A phase I clinical trial of dTCApFs, a derivative of a novel human hormone peptide, for the treatment of advanced/metastatic solid tumors
}

\author{
SALOMON M. STEMMER ${ }^{1,2}$, OFER BENJAMINOV ${ }^{2,3}$, MICHAEL H. SILVERMAN $^{4}$, \\ UZIEL SANDLER $^{4,5}$, OFER PURIM ${ }^{1,2}$, NAOMI SENDER $^{1}$, CHEN MEIR $^{1}$, \\ PNINA OREN-APOTEKER ${ }^{4}$, JOEL OHANA ${ }^{4}$ and YORAM DEVARY ${ }^{4}$
}

\begin{abstract}
${ }^{1}$ Davidoff Center, Rabin Medical Center, Institute of Oncology, Petah Tikva 49414; ${ }^{2}$ Sackler Faculty of Medicine, Tel Aviv University, Tel Aviv 6997801; ${ }^{3}$ Department of Radiology, Rabin Medical Center, Petah Tikva 49414; ${ }^{4}$ Immune System Key (ISK) Ltd., Jerusalem 9746009; ${ }^{5}$ Bioinformatics Department, Lev Academic Center (JCT), Jerusalem 91160, Israel
\end{abstract}

Received March 31, 2017; Accepted September 19, 2017

DOI: $10.3892 /$ mco.2017.1505

\begin{abstract}
The aim of the present phase I first-in-human study was to investigate the safety/efficacy of dTCApFs (a novel hormone peptide that enters cells through the T1/ST2 receptor), in advanced/metastatic solid tumors. The primary objective of this open-label dose-escalation study was to determine the safety profile of dTCApFs. The study enrolled patients (aged $\geq 18$ years) with pathologically confirmed locally advanced/metastatic solid malignancies, who experienced treatment failure or were unable to tolerate previous standard therapy. The study included 17 patients (64\% male; median age, 65 years; $47 \%$ colorectal cancer, $29 \%$ pancreatic cancer). The patients received 1-3 cycles of escalating dTCApFs doses $\left(6-96 \mathrm{mg} / \mathrm{m}^{2}\right)$. The mean number \pm standard deviation of treatment cycles/patient was $3.2 \pm 1.4$; no dose-limiting toxicities were observed up to a dose of $96 \mathrm{mg} / \mathrm{m}^{2}$, and the maximum tolerated dose was not reached. Half-life, maximal plasma concentration, and dTCApFs exposure were found to be linearly correlated with dose. Five patients were treated for $\geq 3$ months $\left(12,24,48 \mathrm{mg} / \mathrm{m}^{2}\right)$ and experienced stable disease throughout the treatment period, and 1 experienced pathological complete response. Analysis of serum biomarkers revealed decreased levels of angiogenic factors at dTCApFs concentrations of $12-48 \mathrm{mg} / \mathrm{m}^{2}$, increased levels of anticancer cytokines, and induction of the endoplasmic reticulum (ER) stress biomarker GRP78/BiP. Efficacy and biomarker data suggest that patients whose tumors were T1/ST2-positive exhibited a better response to dTCApFs. In conclusion,
\end{abstract}

Correspondence to: Professor Salomon M. Stemmer, Davidoff Center, Rabin Medical Center, Institute of Oncology, 39 Jabotinski Street, Petah Tikva 49414, Israel

E-mail: stemmer@post.tau.ac.il; salomon.stemmer@gmail.com

Key words: metastatic cancer, phase 1, solid tumor, T1/ST2, dTCApFs
dTCApFs was found to be safe/well-tolerated, and potentially efficacious, with linear pharmacokinetics. Consistent with preclinical studies, the mechanism through which dTCApFs exerts anticancer effects appears to involve induction of ER stress, suppression of angiogenesis, and activation of the innate immune response. However, further studies are warranted.

\section{Introduction}

dTCApFs (Nerofe ${ }^{\mathrm{TM}}$, Immune System Key Ltd., Jerusalem, Israel) is a novel hormone peptide (14 amino acids) with a demonstrated anticancer activity in the preclinical setting (personal communication with Dr Devary's laboratory, ISK Ltd.). dTCApFs is a derivative of the tumor cell apoptosis factor (TCApF), a 84-amino acid hormone peptide naturally expressed in the thymus, colon and frontal lobe of the brain (1).

Studies in pancreatic, breast and ovarian cell lines investigating the mechanism of action (MOA) through which dTCApFs exerts its anticancer effects, revealed that dTCApFs enters the cells through the T1/ST2 receptor (a member of the Toll/interleukin-1 receptor superfamily), and leads to apoptosis of cancer cells through a novel MOA involving induction of two opposing effects: Induction of structural changes in the Golgi apparatus, loss of Golgi function and induction of endoplasmic reticulum (ER) stress, along with downregulation of the ER stress repair mechanism (personal communication).

We herein report the results of the first-in-human study investigating the safety and efficacy of dTCApFs for the treatment of advanced/metastatic solid tumors.

\section{Patients and methods}

Patients. The present study included adult patients (aged $\geq 18$ years) with pathologically confirmed locally advanced and/or metastatic solid malignancies, who experienced treatment failure or were unable to tolerate previous standard therapy. The key inclusion criteria included evaluable/measurable disease and Eastern Cooperative Oncology Group performance status $\leq 1$. Patients with liver cancer/hepatic 
metastases were considered eligible if liver function met certain criteria, and patients with brain metastases were considered eligible if radiation therapy was completed $\geq 4$ weeks prior to enrollment and the patient received $\leq 4 \mathrm{mg}$ /day of dexamethasone. The key exclusion criteria included receiving anticancer treatment 14 days prior to the initiation of the study drug, and a life expectancy of $<16$ weeks.

Study design. This was a formal open-label phase I dose-escalation study. The primary objective was to determine the maximum tolerated dose (MTD) and safety profile of dTCApFs. Assessments included drug exposure, adverse events (AEs) graded according to the Common Terminology Criteria for Adverse Events, version 4.0 (https://evs.nci.nih. gov/ftp1/CTCAE/CTCAE_4.03_2010-06-14_QuickReference_5x7. pdf), and characterization of dose-limiting toxicities (DLTs). Other objectives included assessment of serum levels of angiogenic factors following dTCApFs administration, pharmacokinetics (PK) and pharmacodynamics (PD) analyses, as well as assessment of receptor staining and tumor response.

The dose escalation study followed a traditional ' $3+3$ ' scheme and included doses of $6,12,24,48$ and $96 \mathrm{mg} / \mathrm{m}^{2}$ of intravenous (IV) dTCApFs, 3 times/week in consecutive 28-day cycles. The patient's allocation is presented in Fig. 1. In all 3-patient cohorts, there was an interval of 2 weeks between the first dose for the first and second patients, and $\geq 1$ week for the third patient. New dose levels started after a follow-up of $\geq 28$ days for the 3 patients at the previous level. MTD was defined as the highest dose level at which $\geq 1$ of the 3 subjects experienced a DLT during their first cycle of treatment. Patients who did not complete their first cycle of treatment for reasons unrelated to AEs were replaced. In addition, PK parameters, including area under the curve (AUC), maximal plasma concentration (Cmax) and plasma half-life (t1/2) were determined. PK parameters were estimated using non-compartmental models.

The clinical activity of dTCApFs was assessed every 8 weeks by physical examination, computed tomography (CT), or magnetic resonance imaging techniques (for evaluable disease only), using the Response Evaluation Criteria In Solid Tumors v1.1 (https://ctep.cancer.gov/protocoldevelopment/docs/recist_guideline.pdf); where appropriate, informative tumor markers were measured in every cycle.

This study was approved by the Institutional Review Board of Rabin Medical Center and the Ministry of Health of Israel, and was conducted at the Davidoff Center, Rabin Medical Center in accordance with the principles of the Declaration of Helsinki. All the patients signed an informed consent prior to enrollment. The study was registered at ClinicalTrials.gov (NCT01690741).

Biomarker analysis. Blood samples were collected from patients and placed on ice for $10 \mathrm{~min}$. Serum samples were collected by centrifugation at $1,000 \mathrm{xg}$ for $10 \mathrm{~min}$ at $4^{\circ} \mathrm{C}$, kept in separate vials at $\leq-20^{\circ} \mathrm{C}$, and shipped to Immune System Key Ltd. at $-20^{\circ} \mathrm{C}$, where they were thawed, aliquoted, and stored at $\leq-20^{\circ} \mathrm{C}$. Repeated freeze-thaw cycles were avoided.

Immunohistochemistry (IHC) staining was performed for T1/ST2 receptor using a full-length anti-ST2 antibody (GenMed, Plymouth, MN, USA). Serum levels of various factors were measured with enzyme-linked immunosorbent assay (ELISA). The measured factors included vascular endothelial growth factor (VEGF), VEGF-D, epidermal growth factor, angiopoietin-1, fibroblast growth factor (FGF)-1, FGF-2, platelet-derived growth factor (PDGF)-AA, PDGF-BB, transforming growth factor (TGF)- $\beta$ (all using ELISA kits by R\&D systems, Abingdon, UK); granulocyte-macrophage colony-stimulating factor (GM-CSF), interleukin (IL)-2, IL-12p70, IL-21 and tumor necrosis factor (TNF)- $\alpha$ (Millipore, Billerica, MA, USA); and glucose-regulated protein 78 (GRP78)/BiP (Enzo, New York, NY, USA).

Statistical analysis. Descriptive statistics were used for all analyses and were performed with $\mathrm{SAS}^{\circledR}$ software, version 9.1 (SAS Institute Inc., Cary, NC, USA). Regression analysis was used to study 2-way correlation between tumor change per month, administered doses of dTCApF, and levels of the ER-stress biomarker (BiP). The statistical significance of the correlation was validated using F-statistics.

\section{Results}

Patients. A total of 39 patients were screened, of whom were 17 enrolled and completed the study. The majority of the patients (64\%) were male, and the median age (range) was 65 (51-94) years. Almost half of the patients (47\%) had colorectal cancer and $29 \%$ had pancreatic cancer. Apart from 1 patient, all other patients had received several lines of anticancer therapy (e.g., chemotherapy, radiotherapy and biological therapy) prior to enrolment (Table I). The patients received 1-3 cycles of escalating dTCApFs doses $\left(6,12,24,48\right.$ and $\left.96 \mathrm{mg} / \mathrm{m}^{2}\right)$, as detailed in Fig. 1.

Safety and tolerability. The mean number of treatment cycles per patient was $3.2 \pm 1.4$. No DLTs were observed in any patient up to cohort 5 . The AEs are summarized in Table II. None were related to the study drug. Hypertension, anemia, vomiting, diarrhea and abdominal pain were the most frequently reported grade $2 \mathrm{AEs}$, and hypertension was the most frequently reported grade $3 \mathrm{AE}$. Vomiting was the only grade $4 \mathrm{AE}$, reported in 1 patient. The majority of the AEs were self-resolving. Overall, treatment with dTCApFs was well-tolerated, with no cumulative toxicity. MTD was not reached.

$P K$ results. The PK results for the first day of cycles 1 and 2 are summarized in Table III; t1/2, Cmax and AUC0 were found to be linearly correlated with dose. Dose-dependent plasma concentrations of dTCApFs were observed (Fig. 2).

Efficacy. Of the 17 patients who were treated for $\geq 3$ months $\left(12,24\right.$ and $\left.48 \mathrm{mg} / \mathrm{m}^{2}\right), 5$ experienced stable disease (SD) throughout the treatment period. Notably, 1 patient was suffering from lower back pain, weakness and referred pain in the left extremity, due to a spinal cord neoplasia compressing the spinal cord (Ki-67, 30\%; ST2-positive staining). Treatment with painkillers (e.g., tramadol, oxycodone/naloxone, morphine and pregabalin) was unsuccessful, and the patient used a walker. After 6 months of treatment (12, 24 and $48 \mathrm{mg} / \mathrm{m}^{2}$ ), the patient's walking improved without the 
Table I. Patient demographics and baseline characteristics.

\begin{tabular}{|c|c|c|c|c|c|}
\hline \multirow[b]{2}{*}{ Characteristics } & \multicolumn{5}{|c|}{ dTCApFs dose, $\mathrm{mg} / \mathrm{m}^{2}$} \\
\hline & $6(n=3)$ & $12(n=3)$ & $24(n=3)$ & $48(n=3)$ & $96(n=5)$ \\
\hline \multicolumn{6}{|l|}{ Age, years } \\
\hline Median (range) & $63(62-77)$ & $61(58-62)$ & $65(57-67)$ & $72(51-94)$ & $64(55-77)$ \\
\hline Mean (SE) & $68(5)$ & $67(4)$ & $67(2)$ & $72(8)$ & $64(9)$ \\
\hline Sex, n (male/female) & $3 / 0$ & $2 / 1$ & $1 / 2$ & $2 / 1$ & $3 / 2$ \\
\hline \multicolumn{6}{|l|}{ Tumor type, $\mathrm{n}$} \\
\hline Colorectal & 3 & 2 & 0 & 2 & 1 \\
\hline Pancreatic & 0 & 0 & 1 & 0 & 4 \\
\hline Other $^{\mathrm{a}}$ & 0 & 1 & 2 & 1 & 0 \\
\hline \multicolumn{6}{|l|}{ Prior therapies, $\mathrm{n}$} \\
\hline Chemotherapy & 3 & 4 & 4 & 1 & 3 \\
\hline Radiotherapy & 1 & 2 & 1 & 1 & 0 \\
\hline Surgery & 2 & 2 & 1 & 1 & 2 \\
\hline Treatment with biological agents & 0 & 0 & 1 & 0 & 0 \\
\hline $\begin{array}{l}\text { Treatment with small molecules, } \\
\text { such as TKIs. }\end{array}$ & 0 & 0 & 0 & 1 & 0 \\
\hline
\end{tabular}

ancludes neoplasms in the small intestine, lung, liver, and spinal cord. SE, standard error; TKIs, tyrosine kinase inhibitors.

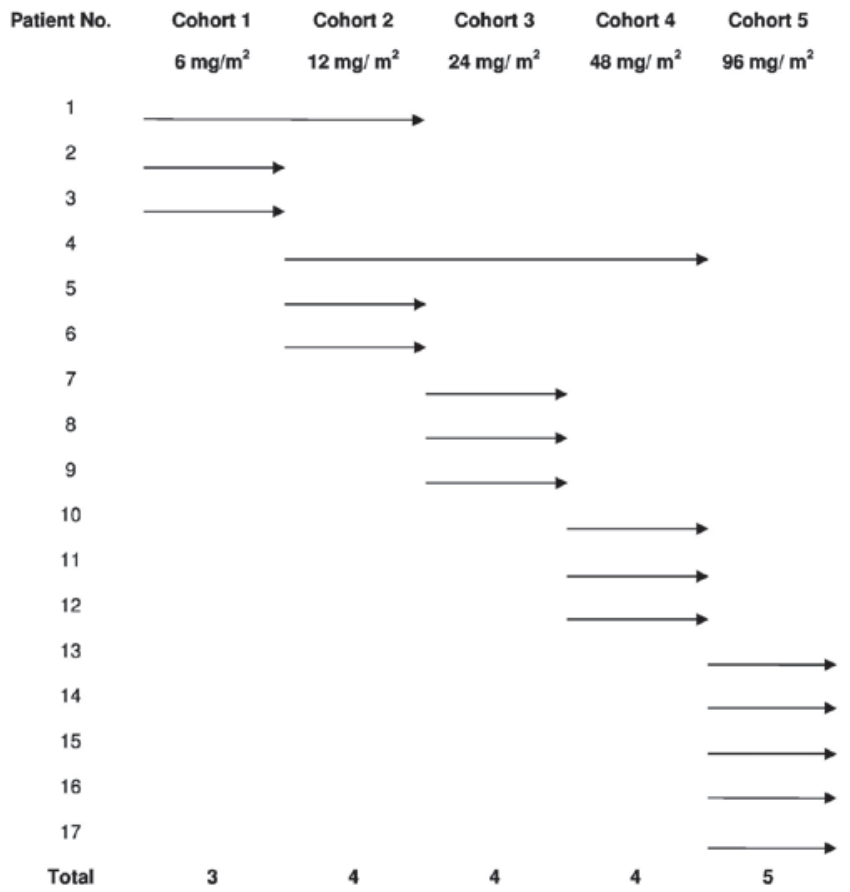

Figure 1. Schematic representation of patient randomization and allocation.

need for any painkiller medication. Surgery was performed after 11 months of treatment. At surgery, no malignancy was observed; however, scar tissue and bleeding were noted, and the histopathological analysis revealed strong presence of natural killer (NK) cells and dendritic cells. A second patient who entered the study with progressive disease after receiving 4 prior lines of chemotherapy treatment, maintained SD through 6 cycles of dTCApFs $\left(6\right.$ and $\left.12 \mathrm{mg} / \mathrm{m}^{2}\right)$.
Progression-free survival (PFS) analysis revealed that 6 patients experienced a longer PFS on dTCApFs compared with their prior regimen, and 1 patient had a PFS that was comparable to that on his prior regimen; 1 patient who had not receive prior treatments was able to remain on the study drug for 330 days (Table IV). A regression analysis [robust regression model $(2,3)]$ computing F statistics P-values revealed a statistically significant correlation between changes in tumor size and the administered dTCApFs doses (Fig. 3).

Biomarker analysis. Treatment with dTCApFs at a dose of $6 \mathrm{mg} / \mathrm{m}^{2}$ led to an increase in serum levels of angiopoietin-1, FGF-1, FGF-2, PDGF-AA, PDGF-BB, VEGF-D, TGF- $\beta$ and VEGF. At doses of $12-48 \mathrm{mg} / \mathrm{m}^{2}$, a decrease in the serum levels of these factors was observed, and at $96 \mathrm{mg} / \mathrm{m}^{2}$, an increase in all factors, except for VEGF-D, was noted. In addition, the serum levels of all anticancer cytokines, such as GM-CSF, IL2, IL-12p70, IL-21 and TNF- $\alpha$, increased with dTCApFs administration in all dose levels (Table V).

To assess the MOA of dTCApFs, patients were examined by their T1/ST2 status, as dTCApFs has been shown to enter the cells through this receptor (personal communication). A total of 14 patients underwent $\mathrm{CT}$ at 8 weeks and were evaluable for this analysis. We observed that patients whose tumors were T1/ST2-positive (by IHC) remained in the trial longer compared with those whose tumors were T1/ST2-negative (Fig. 4A) and experienced SD on dTCApFs treatment. Therefore, the patient population was re-analyzed (changes in tumor size vs. administered dTCApFs dose), after excluding T1/ST2-positive patients $(n=9)$. In this re-analysis, the correlation coefficient increased from 0.56 to 0.76 , and the standard deviation for tumor size decreased from 4.6 to $2.6(\mathrm{P}=0.02)$. A separate statistically valid regression analysis 
Table II. Summary of adverse events by dTCApFs dose group.

dTCApFs dose, $\mathrm{mg} / \mathrm{m}^{2}$

\begin{tabular}{llllll}
\hline Adverse events & $12(\mathrm{n}=3)$ & $24(\mathrm{n}=3)$ & $48(\mathrm{n}=3)$ & $96(\mathrm{n}=5)$ \\
\hline
\end{tabular}

Grade 1

Blood disorders

Anemia

Increased INR

GI disorders

Abdominal pain

Bowel obstruction

Diarrhea

GI hemorrhage

Vomiting

General disorders

Dehydration

Fatigue

Hypertension

Nervous system disorders

Neuropathy

Grade 2

Pain

Pain, leg

Pain, upper back

Respiratory system disorders

Cough

Skin disorders

Pruritus

Urticaria

Hepatic and urinary disorders

ALT increase

AST increase

Bilirubin increase

Liver dysfunction

Urinary tract infection

Grade 3

Blood disorders

Increased INR

General disorders

Hypertension

Hepatic and urinary disorders

Bilirubin increase

GI disorders

Bowel obstruction

Diarrhea

GI hemorrhage

Grade 4

GI disorders

Vomiting
3

0

0

1

0

1

2

0

0

3

0

0

0

0

0

0

0

0

0

0

0

0

0

2

0

1

0

1

$$
0
$$

0

\section{1}

0

2

0

$$
0
$$

\section{0}

1

1

1

02

2

0

1

0

0

0

$$
0
$$

0

2

0

0

0

0

0

0

1

1

0

0

0

0

0

0

0

0

0

0

0

0

0

1

0

0

0

0

0

$$
0
$$

0

1

0

1

1

0

2

0

(20

0
1

0

0

1
0

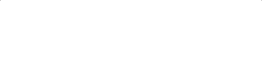

0

(1)

$$
1
$$

$0 \quad 0$

$0 \quad 0$

$\begin{array}{lll}0 & 0 \\ 0 & 0\end{array}$

ALT, alanine transaminase; AST, aspartate aminotransferase; GI, gastrointestinal; INR, international normalized ratio. 
Table III. Pharmacokinetics of dTCApFs on the first day of cycles 1 and 2 (each cycle was 28 days).

\begin{tabular}{|c|c|c|c|c|c|c|c|c|c|c|}
\hline \multirow{2}{*}{$\begin{array}{l}\text { dTCApFs dose, } \\
\mathrm{mg} / \mathrm{m}^{2}\end{array}$} & \multicolumn{5}{|c|}{ Cycle 1 , day 1} & \multicolumn{5}{|c|}{ Cycle 2, day 1} \\
\hline & $6(n=3)$ & $12(\mathrm{n}=4)$ & $24(n=4)$ & $48(n=4)$ & $96(n=3)$ & $6(n=3)$ & $12(n=4)$ & $24(\mathrm{n}=4)$ & $48(n=4)$ & $96(n=3)$ \\
\hline $\mathrm{AUC}_{0}, \mathrm{ng} \cdot \mathrm{h} / \mathrm{ml}$ & 3,813 & 12,905 & 49,630 & 79,935 & 206,742 & 9,719 & 11,452 & 57,069 & 100,093 & 294,682 \\
\hline $\mathrm{C}_{\max }, \mathrm{ng} / \mathrm{ml}$ & 1,209 & 6,048 & 14,609 & 18,267 & 32,964 & 1,536 & 6,048 & 14,609 & 22,113 & 32,016 \\
\hline $\mathrm{t}_{1 / 2}, \mathrm{~h}$ & 2.3 & 2.1 & 3.2 & 4.9 & 6.0 & 2.8 & 2.0 & 3.7 & 4.6 & 8.5 \\
\hline
\end{tabular}

AUC, area under the curve; $\mathrm{C}_{\max }$, maximal plasma concentration; $\mathrm{t}_{1 / 2}$, plasma half-life.

Table IV. PFS on the last regimen before enrolling the study and on dTCApFs.

\begin{tabular}{rrr}
\hline Patient no. & $\begin{array}{c}\text { PFS on the last regimen } \\
\text { pre-enrollment, days }\end{array}$ & $\begin{array}{c}\text { PFS on dTCApFs, } \\
\text { days }\end{array}$ \\
\hline 1 & 480 & 53 \\
2 & 134 & 25 \\
$\mathbf{3}$ & $\mathbf{1 1 0}$ & $\mathbf{1 7 0}$ \\
$\mathbf{4}$ & $\mathbf{0}$ & $\mathbf{3 3 0}$ \\
$\mathbf{5}$ & $\mathbf{5 2}$ & $\mathbf{5 1}$ \\
6 & 384 & 110 \\
7 & $\mathbf{5 4}$ & $\mathbf{9 0}$ \\
8 & 80 & 52 \\
9 & 375 & 60 \\
10 & 1,800 & 14 \\
$\mathbf{1 1}$ & $\mathbf{4 1}$ & $\mathbf{5 2}$ \\
$\mathbf{1 2}$ & $\mathbf{4 2}$ & $\mathbf{5 0}$ \\
13 & 96 & 42 \\
14 & 365 & 40 \\
$\mathbf{1 5}$ & $\mathbf{1}$ & $\mathbf{8 0}$ \\
16 & 105 & 45 \\
17 & 564 & 41 \\
\hline
\end{tabular}

Rows with bold print represent patients who experienced PFS on dTCApFs, which was comparable with or exceeded that of their last regimen pre-enrollment. PFS, progression-free survival.

for the subpopulation of T1/ST2-positive patients could not be performed due to the small sample size $(n=4)$. The serum levels of the GRP78/BiP protein (ER stress biomarker) were also measured prior to the initiation of dTCApFs treatment and after 29 days of treatment. A statistically significant correlation was observed between administered dTCApFs doses and change in serum GRP78/BiP levels $(\mathrm{P} \leq 0.05)$, as well as between changes in tumor size and change in serum GRP78/BiP levels $(\mathrm{P} \leq 0.002)$, suggesting that dTCApFs induced ER stress (Fig. 4B and C). These correlation analyses were then repeated after excluding T1/ST2-negative patients, and an increase in the correlation coefficients (for dTCApFs vs. change in GRP78/BiP levels, from 0.57 to 0.75 ; for change in GRP78/BiP levels vs. changes in tumor size, from 0.79 to 0.83 ) were observed, along with a decrease in the standard deviation for GRP78/BiP changes (from 184 to 67 and from

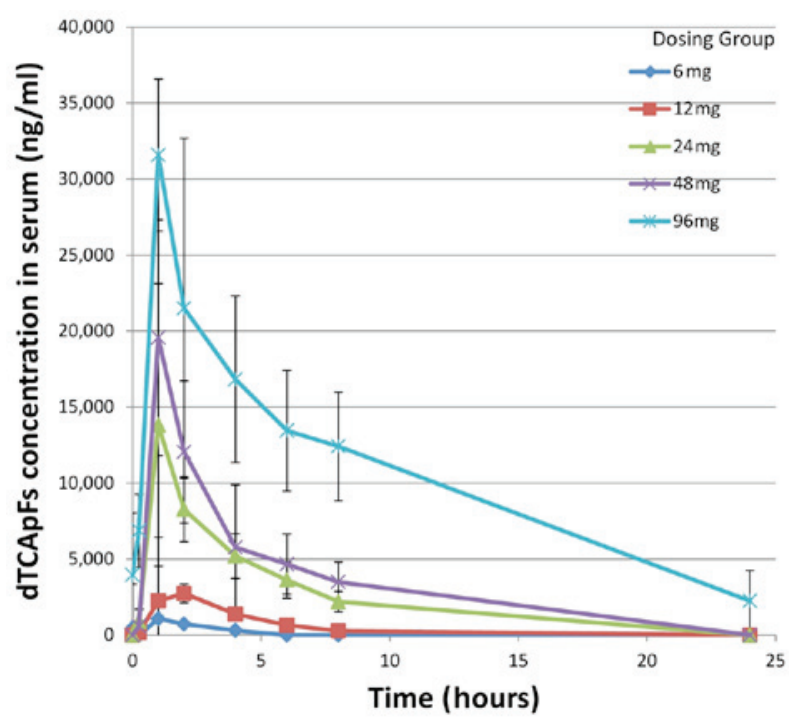

Figure 2. Serum concentrations of dTCApFs over time by dose group. Error bars represent standard deviation.

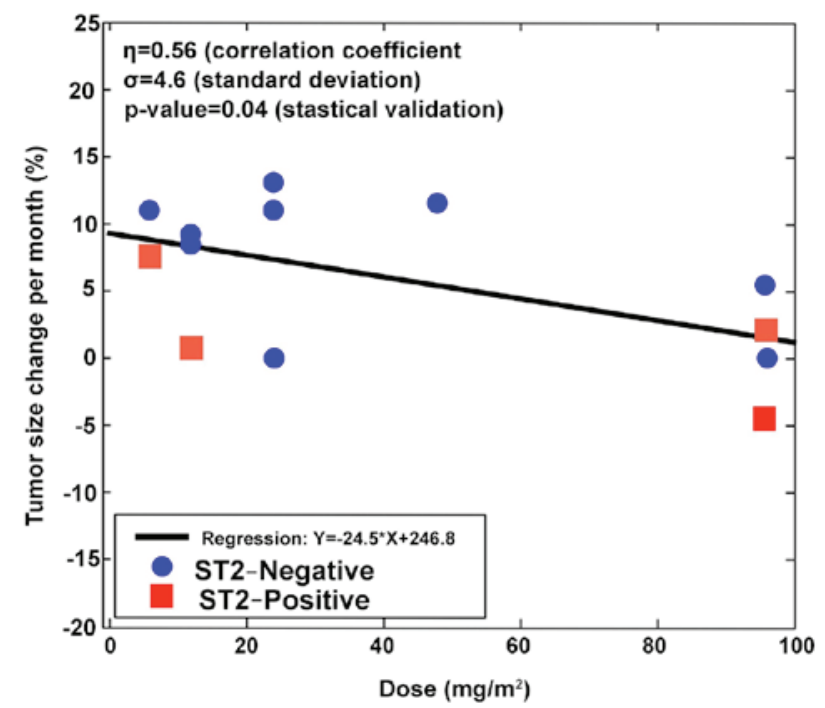

Figure 3. Correlation between changes in tumor size and the administered dTCApFs dose. A total of 14 patients who underwent computed tomography examination at 8 weeks were included in the analysis.

148 to 36 , respectively). These changes were statistically significant $(\mathrm{P} \leq 0.01)$. 
Table V. Mean change (\%) in serum levels of angiogenic factors and cytokines pre- to post- treatment with dTCApFs.

\begin{tabular}{|c|c|c|c|c|c|}
\hline \multirow[b]{2}{*}{ Factors } & \multicolumn{5}{|c|}{ dTCApFs dose, $\mathrm{mg} / \mathrm{m}^{2}$} \\
\hline & $6(n=3)$ & $12(n=3)$ & $24(n=3)$ & $48(n=3)$ & $96(n=5)$ \\
\hline \multicolumn{6}{|l|}{ Angiogenic factors } \\
\hline Angiopoietin-1 & +960 & -80 & -77 & -50 & +70 \\
\hline FGF-1 & +120 & -62 & -20 & -27 & +457 \\
\hline FGF-2 & +199 & -74 & -34 & -13 & +44 \\
\hline PDGF-AA & $+1,379$ & -92 & -79 & -73 & +57 \\
\hline PDGF-BB & $+2,271$ & -95 & -82 & -78 & +185 \\
\hline VEGF-A & +265 & -47 & -62 & -72 & -2 \\
\hline TGF- $\beta 1$ & +18 & -80 & -59 & -20 & No data \\
\hline VEGF-D & +117 & -40 & -54 & -63 & +3 \\
\hline \multicolumn{6}{|l|}{ Cytokines } \\
\hline GM-CSF & $+2,173$ & -97 & +11 & $+5,613$ & +974 \\
\hline IL-12-p70 & +469 & -76 & +83 & +477 & +332 \\
\hline IL-2 & No data & -100 & No data & +242 & +577 \\
\hline IL-21 & +100 & -61 & +84 & $+1,326$ & +29 \\
\hline TNF- $\alpha$ & +4 & -5 & +31 & +74 & +97 \\
\hline
\end{tabular}

FGF, fibroblast growth factor; GM-CSF, granulocyte-macrophage colony-stimulating factor; IL, interleukin; PDGF, platelet-derived growth factor; TGF, transforming growth factor; VEGF, vascular endothelial growth factor; TNF, tumor necrosis factor.

A

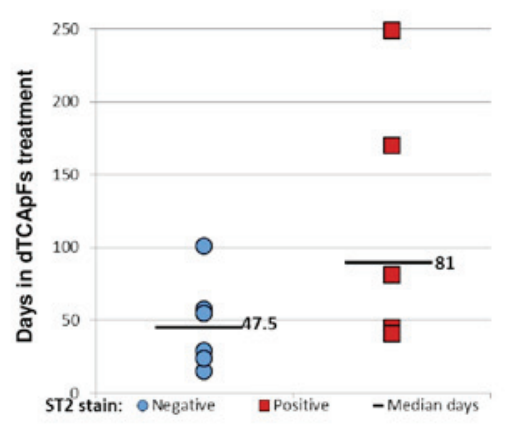

B

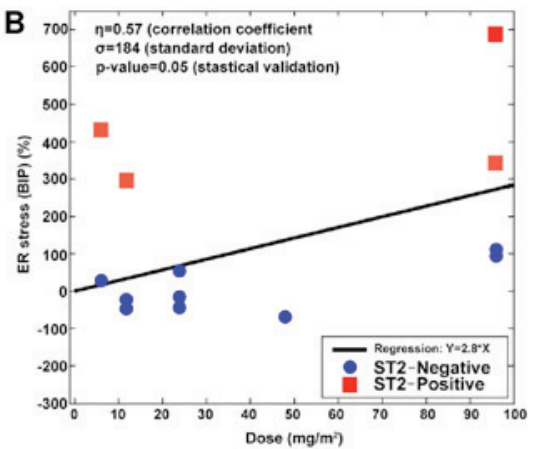

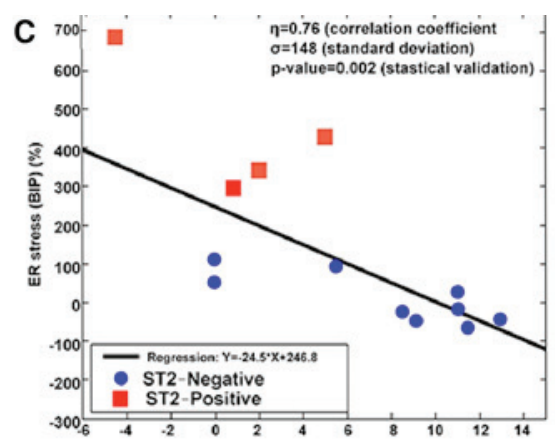

Figure 4. (A) Number of days in trial for patients according to T1/ST2 expression on immunohistochemistry. (B) Correlation between change in serum levels of the endoplasmic reticulum (ER) stress marker BiP and dTCApFs dose. (C) Correlation between change in serum levels of BiP and change in tumor size. A total of 14 patients who underwent computed tomography examination at 8 weeks were included in the analysis.

\section{Discussion}

The aim of the present phase I dose-escalation study was to investigate dTCApFs, a novel hormone peptide, whose activity is driven by its interaction with the T1/ST2 receptor and its anticancer activity is exerted through several MOAs, including a unique MOA involving ER stress induction and downregulation of the ER stress repair mechanism. Intravenous dTCApFs was found to be safe, well-tolerated and potentially efficacious in treating advanced/metastatic solid tumors. Furthermore, the PK examinations revealed that $\mathrm{t} 1 / 2, \mathrm{Cmax}, \mathrm{AUC} 0$ and plasma concentrations of dTCApFs were linearly correlated with dose. In addition, that dTCApFs was found to have anti-angiogenic activity, as well as the ability to induce ER stress and expression of anticancer cytokines.

The present phase I study provides insight into the MOA by which dTCApFs exerts its anticancer effects. dTCApFs enters the cells through the T1/ST2 receptor (personal communication). T1/ST2 is a member of the IL-1 receptor family and IL-33, which regulate the Th1/Th2 immune responses in autoimmune and inflammatory conditions. Lipopolysaccharides have been shown to stimulate T1/ST2 expression in monocytes, muscle cells and splenocytes, both in vitro and in vivo (4). The T1/ST2 receptor is expressed in macrophages, dendritic cells, as well as in mast cells. This receptor is a stable marker of Th2 polarized thymocytes (but not of Th1 polarized thymocytes) and is important in the response of Th2 to viral antigens and allergens (5-7). T1/ST2 has been shown to play an important role in various diseases, including cancer, Alzheimer's disease, inflammatory diseases, trauma, sepsis, cardiovascular diseases and idiopathic pulmonary fibrosis (6-14). Knocking out this receptor in BALB/c mice bearing mammary carcinoma attenuated tumor growth and metastasis. In these knockout mice (compared with wild-type mice), the serum levels of 
IL-17, interferon- $\gamma$ and TNF- $\alpha$ increased, along with higher ex vivo cytotoxic activity of splenocytes, $\mathrm{NK}$ cells and $\mathrm{CD}^{+}$ $\mathrm{T}$ cells (1). In the present study, dTCApFs treatment led to increased serum levels of anticancer cytokines (e.g., GM-CSF, IL-12p70, IL-2, IL-21 and TNF- $\alpha$ ), likely due to the downregulation of the T1/ST2 receptor. In addition, a correlation between the antitumor activity of dTCApFs and T1/ST2 expression status in the tumors was observed. A direct correlation was found between T1/ST2 positivity, tumor size changes and induction of ER stress. These findings are consistent with preclinical studies, where treating ST2 gene knockout OV-90 cells with dTCApFs did not result in ER stress. Taken together, these observations suggest that the T1/ST2 receptor may serve as a biomarker to select T1/ST2-positive patients who are more likely to respond to dTCApFs. Additionally, the biomarker analysis revealed that dTCApFs treatment increased the levels of IL-21 and IL12p70, which are known activators of NK cells $(15,16)$, as well as the levels of GM-CSF and IL-2, which are known activators of dendritic cells $(17,18)$. Furthermore, histopathological analysis of a post-treatment surgical specimen from a patient who achieved a complete response revealed strong presence of NK and dendritic cells. These findings suggest that dTCApFs may activate the innate immune response, consistent with prior studies showing such response with ST2 activation $(19,20)$. Drugs with MOAs involving ST2 activation are currently being investigated $(21,22)$. dTCApFs was also found to have broad anti-angiogenic properties (it reduced the expression of multiple angiogenic factors at levels of $12-48 \mathrm{mg} / \mathrm{m}^{2}$ ). Targeting angiogenesis is a well-established MOA in anticancer drugs, with commercially available and investigational drugs targeting factors such as VEGF and FGF receptors $(5,23-25)$. It should be noted that, despite an observed increase in the levels of angiogenic factors with dTCApFs treatment at the highest investigated dose $\left(96 \mathrm{mg} / \mathrm{m}^{2}\right)$, the cytotoxic activity of dTCApFs was, in fact, enhanced at this dose level, possibly due to another MOA of dTCApFs.

Notably, the findings of dTCApFs-induced ER stress are consistent with our preclinical studies showing a novel mechanism involving two opposing effects of dTCApFs that together result in apoptosis: ER stress induction, and downregulation of the ER stress repair mechanism. Specifically, dTCApFs molecules enter the cells through the ST2 receptor. Subsequently, they bind to the sST2 soluble T1/ST2 receptor, enter the Golgi apparatus, and induce structural changes that lead to destruction of the Golgi apparatus and loss of Golgi function. This, in turn, leads to accumulation of proteins in the ER, resulting in ER stress. dTCApFs also downregulates sXBP1 and, thus, inhibits the ER stress repair mechanism, leading to apoptosis. Interestingly, over the last decade, the interaction between ER stress and tissue vascularization has been intensively investigated and a clear interaction between the stress-response mechanism and VEGF was observed in cancer, diabetic retinopathy, atherosclerosis and ischaemic renal disease (26). GRP78/BiP is as an ER stress marker, and its upregulation following anti-angiogenic therapy has been demonstrated in multiple studies. For example, Han et al, demonstrated that sunitinib treatment, which inhibits PDGF and vascular VEGFR receptors, induced hypoxia in Caki-1 xenografts, that was followed by elevated expression of GRP78/BiP in the treated group compared with the control group (27). It may be hypothesized that dTCApFS interrupts angiogenesis, thereby causing accumulation of unfolded proteins in the ER of the cancer cells, resulting in ER stress, leading to apoptosis.

In conclusion, treatment with intravenous dTCApFs (6-96 mg/m², 3 times/week, in consecutive 28-day cycles) in locally advanced or metastatic solid tumors was found to be safe and well-tolerated, with a dose-dependent, linear PK. dTCApFs suppressed angiogenic factors, induced anticancer cytokine production and ER stress, which likely led to the clinical outcome observed in some of our patients. Positive T1/ST2 staining may serve as a predictive marker for response to dTCApFs. Further studies on the efficacy of dTCApFs in advanced malignancies expressing high levels of T1/ST2 are warranted.

\section{Acknowledgements}

The present study was supported by ISK and Israel Chief Scientist grants (grant no. 54811). Silverman MH, Sandler U, Oren-Apoteker P, Ohana J and Devary Y are employed by ISK.

\section{References}

1. Sandler U, Devary O, Braitbard O, Ohana J, Kass G, Rubinstein AM, Friedman ZY and Devary Y: NEROFE-a novel human hormone-peptide with anti-cancer activity. J Exp Ther Oncol 8: 327-339, 2010.

2. Marazzi A: Algorithms, routines and $\mathrm{S}$ functions for robust statistics: The FORTRAN library ROBETH with an interface to S-PLUS. Chapman and Hall, New York City, NY, 1993.

3. Fox J: Applied regression analysis, linear models, and related methods. Sage Publications, Inc., London, England, 1997.

4. Saccani S, Polentarutti N, Penton-Rol G, Sims JE and Mantovani A: Divergent effects of LPS on expression of IL-1 receptor family members in mononuclear phagocytes in vitro and in vivo. Cytokine 10: 773-780, 1998.

5. Gutheil JC, Campbell TN, Pierce PR, Watkins JD, Huse WD, Bodkin DJ and Cheresh DA: Targeted antiangiogenic therapy for cancer using Vitaxin: A humanized monoclonal antibody to the integrin alphavbeta3. Clin Cancer Res 6: 3056-3061, 2000.

6. Lu DP, Zhou XY, Yao LT, Liu CG, Ma W, Jin F and Wu YF: Serum soluble ST2 is associated with ER-positive breast cancer. BMC Cancer 14: 198, 2014

7. O'Donnell C, Mahmoud A, Keane J, Murphy C, White D, Carey S, O'Riordain M, Bennett MW, Brint E and Houston A: An antitumorigenic role for the IL-33 receptor, ST2L, in colon cancer. Br J Cancer 114: 37-43, 2016.

8. Lambrecht BN, De Veerman M, Coyle AJ, Gutierrez-Ramos JC, Thielemans K and Pauwels RA: Myeloid dendritic cells induce $\mathrm{Th} 2$ responses to inhaled antigen, leading to eosinophilic airway inflammation. J Clin Invest 106: 551-559, 2000.

9. Walzl G, Matthews S, Kendall S, Gutierrez-Ramos JC, Coyle AJ, Openshaw PJ and Hussell T: Inhibition of T1/ST2 during respiratory syncytial virus infection prevents $\mathrm{T}$ helper cell type 2 (Th2)- but not Th1-driven immunopathology. J Exp Med 193: 785-792, 2001.

10. Coyle AJ, Lloyd C, Tian J, Nguyen T, Erikkson C, Wang L, Ottoson P, Persson P, Delaney T, Lehar S, et al: Crucial role of the interleukin 1 receptor family member T1/ST2 in T helper cell type 2-mediated lung mucosal immune responses. J Exp Med 190: 895-902, 1999.

11. Meisel C, Bonhagen K, Löhning M, Coyle AJ, Gutierrez-Ramos JC, Radbruch A and Kamradt T: Regulation and function of T1/ST2 expression on CD4+ T cells: Induction of type 2 cytokine production by T1/ST2 cross-linking. J Immunol 166: 3143-3150, 2001.

12. Xu D, Chan WL, Leung BP, Huang FP, Wheeler R, Piedrafita D, Robinson JH and Liew FY: Selective expression of a stable cell surface molecule on type 2 but not type 1 helper T cells. J Exp Med 187: 787-794, 1998. 
13. Townsend MJ, Fallon PG, Matthews DJ, Jolin HE and McKenzie AN: T1/ST2-deficient mice demonstrate the importance of T1/ST2 in developing primary T helper cell type 2 responses. J Exp Med 191: 1069-1076, 2000.

14. Xiong Z, Thangavel R, Kempuraj D, Yang E, Zaheer S and Zaheer A: Alzheimer's disease: Evidence for the expression of interleukin-33 and its receptor ST2 in the brain. J Alzheimers Dis 40: 297-308, 2014.

15. Van Elssen CH, Vanderlocht J, Frings PW, Senden-Gijsbers BL, Schnijderberg MC, van Gelder M, Meek B, Libon C, Ferlazzo G, Germeraad WT and Bos GM: Klebsiella pneumoniae-triggered DC recruit human NK cells in a CCR5-dependent manner leading to increased CCL19-responsiveness and activation of NK cells. Eur J Immunol 40: 3138-3149, 2010.

16. Frederiksen KS, Lundsgaard D, Freeman JA, Hughes SD, Holm TL, Skrumsager BK, Petri A, Hansen LT, McArthur GA, Davis ID and Skak K: IL-21 induces in vivo immune activation of NK cells and CD8(+) T cells in patients with metastatic melanoma and renal cell carcinoma. Cancer Immunol Immunother 57: 1439-1449, 2008

17. Wuest SC, Edwan JH, Martin JF, Han S, Perry JS, Cartagena CM, Matsuura E, Maric D, Waldmann TA and Bielekova B: A role for interleukin-2 trans-presentation in dendritic cell-mediated $\mathrm{T}$ cell activation in humans, as revealed by daclizumab therapy. Nat Med 17: 604-609, 2011.

18. van de Laar L, Coffer PJ and Woltman AM: Regulation of dendritic cell development by GM-CSF: Molecular control and implications for immune homeostasis and therapy. Blood 119: 3383-3393, 2012.

19. Rank MA, Kobayashi T, Kozaki H, Bartemes KR, Squillace DL and Kita H: IL-33-activated dendritic cells induce an atypical TH2-type response. J Allergy Clin Immunol 123: 1047-1054, 2009.

20. Nabekura T, Girard JP and Lanier LL: IL-33 receptor ST2 amplifies the expansion of NK cells and enhances host defense during mouse cytomegalovirus infection. J Immunol 194: 5948-5952, 2015.
21. ClinicalTrials.gov: Description of a trial investigating Interleukin-12 in ovarian epithelial cancer or primary peritoneal cancer. Available at: https://clinicaltrials.gov/ct2/show/NCT0001 6289?term=IL12p70\&rank=7. Accessed January 11, 2017.

22. ClinicalTrials.gov: Description of the recombinant interleukin-21 in metastaticmelanomaandkidneycancer.Availableat:https://clinicaltrials.gov/ct2/show/NCT00095108?term=IL-21\&rank=7.Accessed January 11, 2017.

23. Vasudev NS and Reynolds AR: Anti-angiogenic therapy for cancer: Current progress, unresolved questions and future directions. Angiogenesis 17: 471-494, 2014.

24. Boehm T, Folkman J, Browder T and O'Reilly MS: Antiangiogenic therapy of experimental cancer does not induce acquired drug resistance. Nature 390: 404-407, 1997.

25. Jain RK: Antiangiogenic therapy for cancer: Current and emerging concepts. Oncology (Williston Park) 19 (4 Suppl 3): S7-S16, 2005.

26. Tahergorabi $\mathrm{Z}$ and Khazaei $\mathrm{M}$ : Imbalance of angiogenesis in diabetic complications: The mechanisms. Int J Prev Med 3: 827-838, 2012 .

27. Han KS, Li N, Raven PA, Fazli L, Frees S, Ettinger S, Park KC, Hong SJ, Gleave ME and So AI: Inhibition of endoplasmic reticulum chaperone protein glucose-regulated protein 78 potentiates anti-angiogenic therapy in renal cell carcinoma through inactivation of the PERK/eIF $2 \alpha$ pathway. Oncotarget 6 : 34818-34830, 2015.

This work is licensed under a Creative Commons Attribution-NonCommercial-NoDerivatives 4.0 International (CC BY-NC-ND 4.0) License. 